\title{
The RNA-seq transcriptome analysis identified genes related to rice seed dormancy
}

\author{
K. XIE ${ }^{1}$, J. BAI ${ }^{1}$, Y.Y. YANG ${ }^{1}$, N.B. DUAN ${ }^{1}$, Y.M. MA ${ }^{1}$, T. GUO ${ }^{1}$, F.Y. YAO ${ }^{2}$, and H.F. DING ${ }^{1,3 *}$ \\ Shandong Center of Crop Germplasm Resources, Jinan 250100, Shandong, P.R. China ${ }^{1}$ \\ Biotechnology Research Center, Shandong Academy of Agricultural Sciences, \\ Jinan 250100, Shandong, P.R. China ${ }^{2}$ \\ College of Life Science, Shandong Normal University, Jinan 250014, Shandong, P.R. China ${ }^{3}$
}

\begin{abstract}
Plant hormones play important roles in seed dormancy and dormancy breaking. We measured the hormone content in rice (Oryza sativa L. cv. Nona Bokra) seeds at different stages and with or without imbibition treatment. We identified 1265 differentially expressed genes (DEGs) between dormant and dormancy-broken seeds using RNA-seq analysis: 1015 genes were significantly up-regulated, while 250 genes were significantly down-regulated. Sixteen DEGs were selected as related to seed dormancy, and their expressions were validated using quantitative PCR. Three DEGs were in the same position as two reported dormancy QTLs, suggesting that they may be candidate genes that control the dormancy of rice seeds. Our study provides an important basis for cloning genes in dormant rice seeds and provides theoretical support for the study of the dormancy mechanism.
\end{abstract}

Additional key words: differentially expressed genes, Oryza sativa, transcription factors.

\section{Introduction}

Seed dormancy is defined as the failure to geminate under favorable environmental conditions, and it is an important agronomic trait which strongly affects rice grain yield and quality (Ogbonnaya et al. 2008). Besides, it is a complex trait as it is determined by the interplay of a gene network and environmental factors (Wang et al. 2014).

Phytohormones play key roles in the induction and maintenance of seed dormancy. Abscisic acid (ABA) promotes the synthesis of seed storage proteins and lipids enhancing desiccation tolerance and dormancy (Finkelstein et al. 2002). In contrast, gibberellic acid (GA) stimulates the growth of seed embryos and softens the surrounding tissues. The ABA/GA balance appears to serve as a primary determinant of seed dormancy/ germination. In the early stage of seed development, a high $\mathrm{ABA} / \mathrm{GA}$ ratio is established and maintained for inhibition of germination and induction of maturation (Liu et al. 2014). During ripening Arabidopsis and barley seeds, accumulation of GA occurs and ABA content decreases.
Abscisic acid suppresses the expression of GA20 oxidase in the GA-biosynthetic pathway. Therefore, it functions as a germination-inhibitor (Nambara and Marion-Poll 2003). The antagonism of ABA and GA is mainly manifested in two aspects. On the one hand, distinct transcription factors control the accumulation of ABA and GA to be in metabolic homeostasis; on the other hand, components of the ABA and GA signal transduction pathway interact (Liu and Hou 2018). Two APETALA 2 (AP2)-domain containing transcription factors play key roles in the antagonism between ABA and GA (Shu et al. 2018).

The auxin participates in a diverse range of processes in plant intrinsic developmental programs and in response to external stimuli (Zhao 2010). In spite of the crosstalk between auxin and ABA, auxin on its own is not considered as a primary modulator of seed germination (Wang et al. 2011). However, previous studies on wheat reported the negative effect of indole-3-acetic acid (IAA) on seed germination and pre-harvest sprouting (Ramaih

Submitted 28 March 2018, last revision 4 September 2018, accepted 13 September 2018.

Abbreviations: ABA - abscisic acid; DAH - days after heading; DEGs - differentially expressed genes; DREB - dehydration responsive element binding; GA - gibberellic acid; IAA - indole-3-acetic acid; QTL - quantitative trait locus; qPCR - quantitative PCR; UBC ubiquitin C; UPLC-MS - ultra-performance liquid chromatography coupled with mass spectrometry.

Acknowledgments: This work was supported by the Natural Science Foundation of Shandong Province, China (Grant No. ZR2012CQ023), the National Key Research and Development Plan Project (Grant No. 2016YFD0100101-14), and the Doctoral Fund of Shandong Province (Grant No.BS2013NY011).

* Corresponding author; fax: (+86) 531 66659900, e-mail: dinghf2005@163.com 
et al. 2003). Liu (2013) suggested that dormancy breakdown resulting from an after-ripening treatment is due to a reduced sensitivity to auxin. Further investigation incorporating genetic and biochemical approaches have uncovered $\mathrm{ABI} 3$ as an essential component in auxinmediated seed dormancy and germination.

\section{Materials and methods}

Plants: Seeds of indica type rice (Oryza saliva L.), cv. Nona Bokra display a strong dormancy with a germination rate lower than $2 \%$. All plants were grown in a greenhouse of Shandong Academy of Agricultural Sciences (Jinan, China). Nona Bokra was grown in normal conditions for nearly two months and then transferred to the short day (9-h photoperiod) to promote heading. The heading date of each individual was marked by the emergence of the first panicle from the leaf sheath. The seeds at 20 and $35 \mathrm{~d}$ after heading (DAH) were harvested.

A part of $35 \mathrm{DAH}$ seeds were stored at $4{ }^{\circ} \mathrm{C}$ to maintain their dormancy (dormant seeds) while other seeds were heated at $50{ }^{\circ} \mathrm{C}$ for $7 \mathrm{~d}$ to break dormancy (dormancybroken seeds). Dormant and dormancy-broken seeds were used for germination test. Dormancy was evaluated following the method described by Wan (1997). Samples (50 seeds in each sample, 3 replications per treatment) were laid on doubled sheets of moistened filter paper in a 9-cm Petri dish maintained in the dark at a temperature of $30{ }^{\circ} \mathrm{C}$ and a $100 \%$ relative humidity for $7 \mathrm{~d}$. The emergence of the radicle and/or plumule denoted a broken dormancy.

Hormone detection: DAH seeds 20 and $35 \mathrm{~d}$ after heading, dormancy-broken seeds, and dormancy-broken seeds imbibed for $48 \mathrm{~h}$ were used for hormone detection, each with 3 biological replicates. Hormone extraction and detection were carried out by the Public Analysis and Testing Center of Beijing Forestry University using ultra-performance liquid chromatography coupled with mass spectrometry (UPLC-MS).

RNA-Seq analysis and sequence annotations: To understand the molecular basis of seed dormancy in rice, we built high-throughput sequencing libraries for dormant and dormancy-broken seeds, which were immersed in water for $48 \mathrm{~h}$, each with three biological replicates. Total RNA was extracted using a Trizol reagent (Invitrogen, Carlsbad, CA, USA) following the manufacturer's procedures. The quantity and purity of total RNA were analyzed by a Bioanalyzer 2100 and RNA 6000 Nano Lab Chip kit (Agilent, Palo Alto, CA, USA) with RNA integrity number $(\mathrm{RIN})$ number $>7$. 0. PolyA mRNA was
The aim of these experiments was to determine hormone content in rice seeds at four different growth stages and then to analyze the transcriptome sequencing data generated from dormant seeds and dormancy-broken seeds, and to estimate differently expressed genes.

purified from approximately $10 \mu \mathrm{g}$ of total RNA using magnetic beads coupled with poly-T oligo (Invitrogen). After purification, the mRNA was exposed to divalent cations under an elevated temperature for fragmentation. The fragments yielded were reverse-transcribed to create the cDNA library using an mRNA-seq sample preparation kit (Illumina, San Diego, USA), with a $300 \pm 50$ bp average insert size. Then, we performed paired-end sequencing on the Hiseq2000 (Illumina, San Diego, CA, USA) in accordance with the vendor's recommended protocol. Obtained RNA-seq reads were cleaned and assembled into unigene sequences.

Unigene sequences were blasted (BLASTx, E-value < $0.00001)$ against existing protein databases including $N R$ (the non-redundant protein sequences database; ftp://ftp.ncbi.nlm.nih.gov/blast/db/), Pfam (the protein families database; http://pfam.xfam.org/), JGI (joint genome institute; http://jgi.doe.gov/), KEGG (Kyoto encyclopedia of genes and genomes; https://www. genome.jp/kegg/) to predict their functions. Cufflinks were used to process the aligned fragments to quantify the abundance of the transcripts. The expressions of all unigenes were represented by fragment per kilobase of exon per million fragments mapped. Based on expression, significant differentially expressed transcripts were identified with $P$-value $\leq 0.05$ and $\log 2$ fold-change $(\log 2 \mathrm{FC}) \geq 1$. The cluster of differentially expressed transcripts was performed using the common perl and $\mathrm{R}$ scripts.

Quantitative real-time polymerase chain reaction: The expressions were confirmed by real-time quantitative (q) PCR analysis using three technical replicates for samples used for RNA-seq assay. Total RNA was isolated, and the first-strand cDNA was synthesized from $1 \mu \mathrm{g}$ of total RNA using a TUREscript $1^{\text {st }}$ Strand cDNA synthesis kit (Aidlab, Beijing, China) following the manufacturer's instructions. The resulting cDNAs were amplified on a qTOWER2.2 system (Analytik, Jena, Germany) using transcript-specific primers (Table 1 Suppl.). Ubiquitin C (UBC) was chosen as a reference gene for normalizing qPCR data. In order to obtain the best PCR result, the concentration of primers and annealing temperature were optimized.. 


\section{Results}

Nona Bokra showed a strong dormancy with a germination rate of $1 \pm 0.33 \%$. A part of $35 \mathrm{DAH}$-seeds was stored at $4{ }^{\circ} \mathrm{C}$, and their germination rate was $1.56 \pm 0.83 \%$. A part of $35 \mathrm{DAH}$-seeds was treated at $50{ }^{\circ} \mathrm{C}$ for $7 \mathrm{~d}$, and their germination rate increased to $98 \pm 0.67 \%$. Hence, the heat treatment was an effective method for breaking rice seed dormancy. On the contrary, storage at $4{ }^{\circ} \mathrm{C}$ was useful for maintaining rice seed dormancy.

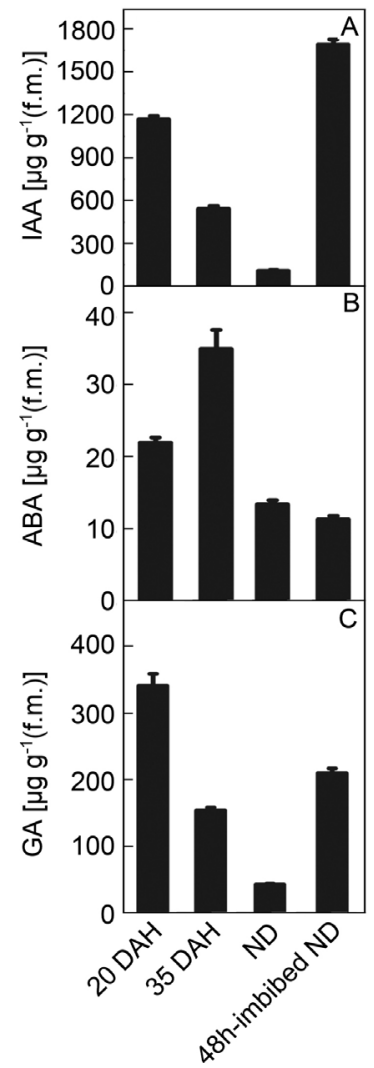

Fig. 1. Content of indole-3-acetic acid (IAA; $A$ ), abscisic acid $(\mathrm{ABA} ; B)$, and gibberellic acid $(\mathrm{GA} ; C)$ in different seeds. Means $\pm \mathrm{SE}, n=3$. DAH - day after heading, ND - dormancy-broken seeds.

We measured the amounts of ABA, GA, and IAA in seeds collected at 20 and 35 DAH. During seed maturation, we detected an increased content of $\mathrm{ABA}$ and a decreased content of IAA and GA (Fig. 1). However, the IAA content was still rather high. The calculated ratio of $\mathrm{ABA} / \mathrm{GA}$ increased as well. After maturation, seed dormancy was broken by heat treatment, and then the effect of imbibition was detected. Compared to the control (imbibition $0 \mathrm{~h}$ ), the content of ABA decreased after imbibition for $48 \mathrm{~h}$, whereas the content of IAA and GA increased. The ratio of ABA to GA decreased. These results suggest that hormones played pivotal roles in the formation and maintenance of seed dormancy (Fig. 1).

In this study, 56476706 and 47695070 raw reads were obtained from imbibed dormant rice seeds and imbibed dormancy-broken seeds, respectively. After a stringent quality assessment and data filtering, high-quality cleaned sequences were then de novo assembled into the reference genome, and 33325409 (84.07\%) and 29978527 (89.08\%) unique mapped reads were obtained, respectively (Table 1).

Table 1. Read numbers based on RNA-seq data. Comparison of dormant seeds and seeds with broken dormancy. They were imbibed for $48 \mathrm{~h}$.

\begin{tabular}{lcc}
\hline Sample & Dormant seeds & $\begin{array}{l}\text { Dormancy-broken } \\
\text { seeds }\end{array}$ \\
\hline Raw reads & 56476706 & 47695070 \\
Clean reads & 56379506 & 47596048 \\
Mapped reads & 39640161 & 33652721 \\
Unique mapped reads & 33325409 & 29978527 \\
Multi mapped reads & 6314752 & 3674194 \\
Pair-end mapped reads & 18183918 & 15510885 \\
Total mapped [\%] & 70.31 & 70.70 \\
Unique mapped [\%] & 84.07 & 89.08 \\
\hline
\end{tabular}

Based on the RNA-seq data of imbibed dormant seeds and imbibed dormancy-broken seeds, we examined differentially expressed genes. In the total of 31511 unigenes, 1265 genes were expressed differently between the two types of seeds. Among them, 1015 genes were significantly up-regulated in dormancy-broken seeds, whereas 250 genes were significantly down-regulated. The gene ontology function enrichment analysis provided the possible biological functions of these DEGs, and 1265 genes were classified into 3 categories: biological processes, cellular components, and molecular functions. In biological processes, the major subcategories were classified as metabolic processes (772 genes), cellular processes ( 720 genes), biosynthetic processes (537 genes), and biological processes (501 genes). Among cellular components, cell components (577 genes) and membranes (450 genes) represented the major subcategories. Protein binding (524 genes) and catalytic activity (414 genes) were the major subcategories in molecular functions (Fig. 2). Their putative contributions to biochemical metabolic pathways and signal transduction cascades were implicated by $K E G G$ enrichment analysis and functional annotation analysis. Out of 1265 genes, 390 genes were assigned to $K E G G$ pathways. Three largest pathway groups were ribosome $(26,6.6 \%)$, starch and sucrose metabolisms (20, $5.1 \%)$, and purine metabolism (15, $3.8 \%)$ (Fig. 1 Suppl.).

According to the functional annotation analysis and gene ontology annotations derived from RNA-seq data, we selected 16 genes that related to seed dormancy from the 1265 DEGs. Then, we assessed their expressions in imbibed dormant seeds and imbibed dormancy-broken seeds via real-time qPCR. The results show that the 
expressions of the 16 genes were similar to those obtained from RNA-seq (Fig. 3) indicating that the RNA-seq was reliable. In dormancy-broken seeds, 11 genes were up-regulated and 5 genes were down-regulated. These genes encode some enzymes, transcription factors, and receptors related to GA, ABA, IAA, and starch (Table 2 Suppl.). They mainly included ABA receptors, GA-20 oxidase, transcription factor GAMYB, auxin-responsive protein, auxin transporter, $\beta$-amylase, $\alpha$-amylase, and AP2 domain containing protein, dehydration-responsive element-binding protein, etc. Therefore, we hypothesize that heat treatment preceding dormancy release may act through its influence on hormone homeostasis.

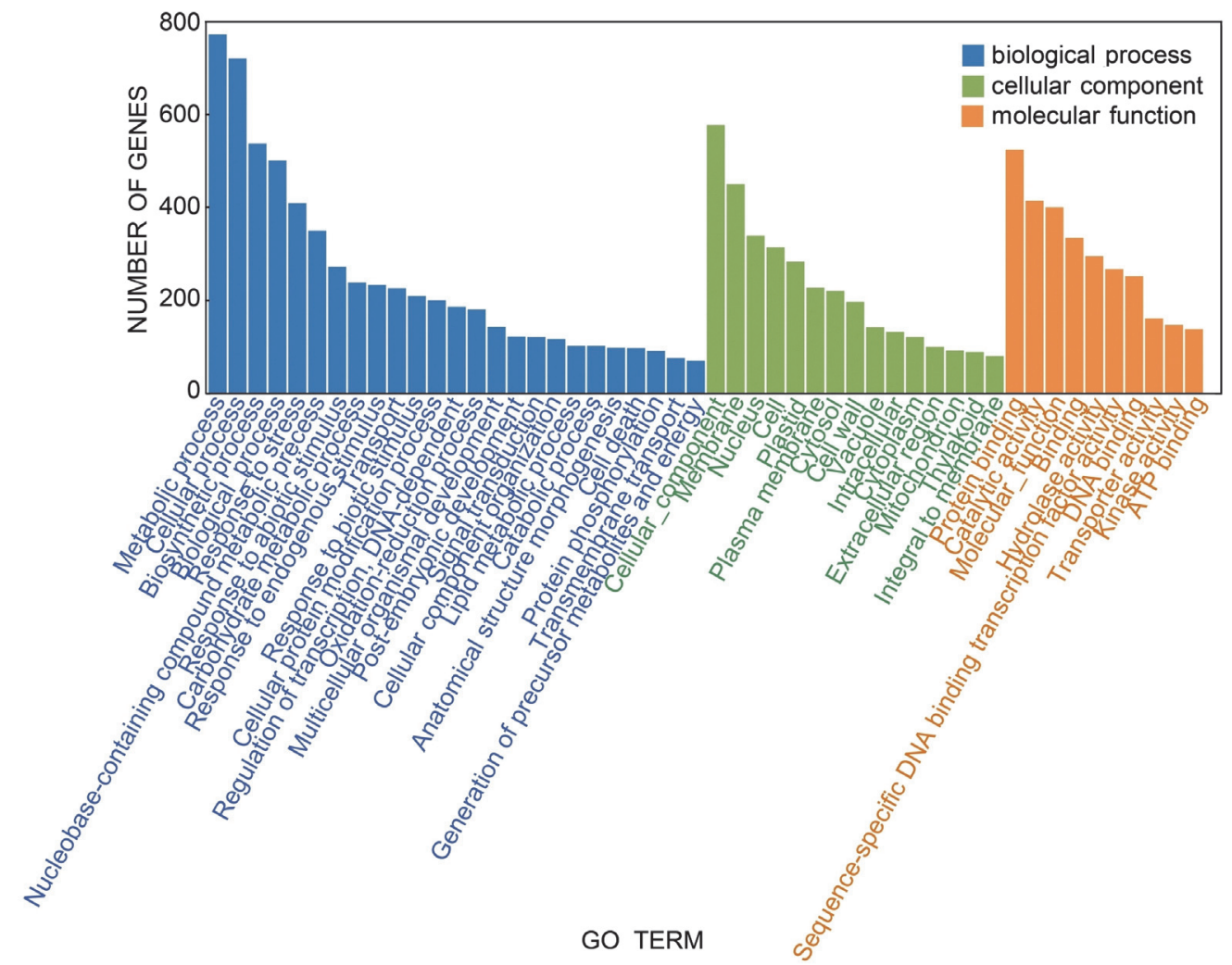

Fig. 2 Enriched gene ontology $(G O)$ terms of differentially expressed genes (DEGs) between dormant seeds and dormancy-broken seeds, both imbibed for $48 \mathrm{~h}$.

\section{Discussion}

Recent studies have revealed a threshold content of ABA which blocks viviparous germination of developing rice seeds (Fang et al. 2008). In general, GA and ABA antagonize in regulating seed dormancy and germination. In agreement with that, the ABA content in rice seeds at 35 DAH was significantly higher than that at $20 \mathrm{DAH}$, whereas the GA and IAA content showed an opposite trend A high content of GA (close to $10^{-4} \mathrm{M}$ ) can be effective in overcoming dormancy, however, there is no evidence that GA content or signaling components change during dormancy release (Bewley 1997). Emerging genetic evidence uncovered the coordination between IAA and ABA in germination inhibition and dormancy preservation (Liu et al. 2013). Abscisic acid represses embryonic axis elongation during seed germination partially through augmenting auxin signaling (Belin et al. 2009). Conversely, Bialek (1992) detected an IAA peak preceding the initiation of root elongation and during imbibition-triggered initial seed swelling. Moreover, an elevated IAA synthesis was found to be coupled with the time of germination (Bialek et al. 1992). Exogenous GA up-regulates the expressions of several auxin-related genes (Ogawa et al. 2003) including both auxin carrier genes and biosynthetic genes. This suggests that GA might also function at least partly through auxin synthesis or transport during Arabidopsis seed germination. Regarding the water imbibition, the ABA content in 48 h-imbibed dormancy-broken seeds was significantly lower than in non-imbibed ones. Conversely, 48-h imbibition resulted in an increased content of IAA and GA in dormancy-broken seeds. There may be a complex and huge coordinating network of IAA, ABA, and GA signalling during the formation, maintenance, and release of seed dormancy and during the seed germination.

Then, the transcriptomes of dormant and dormancy-broken seeds, which were subjected to 
imbibition for $48 \mathrm{~h}$, were analyzed, and the differences of gene expression patterns were studied. As water absorption activates metabolic processes (Bewley 1997), both the types of seeds resumed their metabolic activities after $48 \mathrm{~h}$ of imbibition, the only difference being in their capability to germinate.

Dehydration responsive element binding (DREB) proteins play important roles in plant responses to abiotic stresses as a subgroup of the AP2/EREBP transcription factors (Gutterson and Reuber 2004). Increasing evidence shows that some DREBs are involved in both ABA signaling and stress-responsive pathways (Knight et al. 2004, Sakuma et al. 2006). An ARAG1, an ABA-responsive DREB gene, has been demonstrated to play a role in seed germination and drought tolerance of rice (Zhao et al. 2010). In our study, we found two DREBs (Os08g45110 and Os09g35030). Whether the DREBs surely affect rice seed dormancy and germination, and through which pathway, it remains to be clarified.

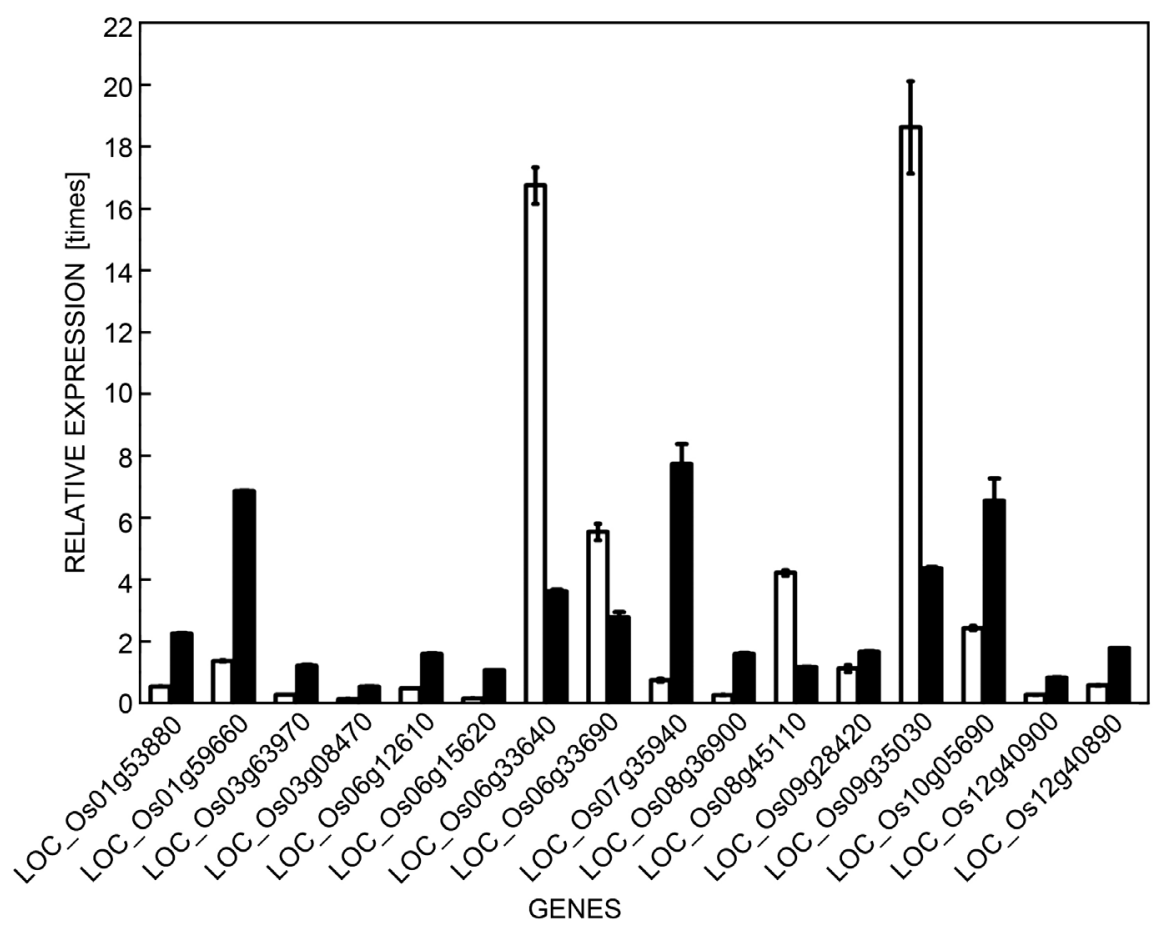

Fig. 3 Verification of RNA-seq results by real-time quantitative PCR. Relative expressions of 16 genes in dormant seeds (white columns) and dormancy-broken seeds (black columns) after imbibition for $48 \mathrm{~h}$. Means \pm SEs, $n=3$.

So far, there are only three genes cloned from the QTLS for rice seed dormancy, Sdr4, qSD7-1, and qSD1-2 (Sugimoto et al. 2010, Gu et al. 2011, Ye et al. 2015). The Sdr4 codes a novel protein with no homology to known proteins. It is proposed to function as a dormancy specific regulator downstream of a global regulator OsVP1 (Sugimoto et al. 2010). The $q S D 7-1$ is a transcription factor gene known to promote the expression of ABA-biosynthetic genes. The $q S D 1-2$ is identified as a OsGA20ox2 locus by map-based cloning and mutant analysis. It is believed that its action in dormancy regulation is a link with a GA-regulated dehydration mechanism. In our study, we found Os03g63970 as GA20 oxidase 1, a key enzyme for GA biosynthesis. We hypothesize that it may be a candidate gene that control rice seed dormancy.

In QTL analyses performed by Marzougui (2012), chromosome segment substitution lines were first made by crossing Nona Bokra and Koshihikari, which show strong and weak dormancy features, respectively. Continued studies on the F2 cross between chromosome segment substitution lines and the original parent Koshihikari mapped three putative QTLs on chromosomes: Sdr6 on chromosome 1 and $S d r 9$ and $S d r 10$ on chromosome 6. In our study, DEGs were mainly associated with ABA, GA, and IAA biosyntheses and signaling pathways. According to our data, Os06g15620, annotated to encode a GA-regulated GASA/GAST/Snak family protein, may well correspond to the QTL $S d r 9$. The Os06g33640 and Os $06 g 33690$ were implicated to encode ABA receptors, and the loci of Os06g33640 and Os06g33690 are similar to the location of Sdr10. In Marzougui (2012) and our study, we all took Nona Bokra as the research object. So our study may help to predict the functions of $S d r 9$ and/or Sdr10. In our study, we didn't detect DEGs corresponded to the QTL Sdr6 which was detected by Marzougui (2012). The failure in detection likely resulted from environmental conditions since the expression of $S d r 6$ is strongly affected by factors such as temperature and moisture.

Seed dormancy is the integrated result of an optimum balance between various phytohormones and environmental factors. In the future, we will research the detailed 
functions of 16 genes found in our study that related to the formation and maintenance of rice seed dormancy. We hope that our future research could provide new candidate genes for breeding rice cultivars harboring moderate dormancy, and explain the mechanisms underlying dormancy formation and maintenance in rice seeds.

\section{References}

Belin, C., Megies, C., Hauserova, E., Lopez-Molina, L.: Abscisic acid represses growth of the Arabidopsis embryonic axis after germination by enhancing auxin signaling. - Plant Cell 21: 2253-2268, 2009.

Bewley, J. D.: Seed germination and dormancy. - Plant Cell 9: 1055-1066, 1997.

Bialek, K., Michalczuk, L.,Cohen, J. D.: Auxin biosynthesis during seed germination in Phaseolus vulgaris. - Plant Physiol 100: 509-517, 1992.

Fang, J., Chai, C., Qian, Q., Li, C., Tang, J., Sun, L., Huang, Z., Guo, X., Sun, C., Liu, M., Zhang, Y., Lu, Q., Wang, Y., Lu, C., Han, B., Chen, F., Cheng, Z., Chu, C.: Mutations of genes in synthesis of the carotenoid precursors of ABA lead to pre-harvest sprouting and photo-oxidation in rice. - Plant $\mathrm{J}$. 54: 177-189, 2008.

Finkelstein, R.R., Gampala, S.S.,Rock, C.D.: Abscisic acid signaling in seeds and seedlings. - Plant Cell 14 (Suppl): S15-S45, 2002.

Gu, X.Y., Foley, M.E., Horvath, D.P., Anderson, J.V., Feng, J., Zhang, L., Mowry, C.R., Ye, H., Suttle, J.C., Kadowaki, K.,Chen, Z.: Association between seed dormancy and pericarp color is controlled by a pleiotropic gene that regulates abscisic acid and flavonoid synthesis in weedy red rice. - Genetics 189: 1515-1524, 2011.

Gutterson, N., Reuber, T.L.: Regulation of disease resistance pathways byAP2/ERF transcription factors. - Curr. Opin. Plant Biol. 7: 465-471, 2004.

Knight, H., Zarka, D.G., Okamoto, H., Thomashow,M.F., Knight,M.R.: Abscisic acid induces $C B F$ gene transcription and subsequent inductionof cold-regulated genes via the CRT promoter element. - Plant Physiol. 135: 1710-1717, 2004.

Liu, A., Gao, F., Kanno, Y., Jordan, M.C., Kamiya, Y., Seo, M., Ayele, B T.: Regulation of wheat seed dormancy by after-ripening is mediated by specific transcriptional switches that induce changes in seed hormone metabolism and signaling. - PLoS ONE 8: e56570, 2013.

Liu, X., Zhang, H., Zhao, Y., Feng, Z., Li, Q., Yang, H.Q., Luan, S., Li, J., He, Z.H.: Auxin controls seed dormancy through stimulation of abscisic acid signaling by inducing ARF-mediated ABI3 activation in Arabidopsis. - Proc. nat. Acad. Sci. USA 110: 15485-15490, 2013.

Liu, X., Hou, X.L.: Antagonistic regulation of ABA and GA in metabolism and signaling pathways. - Front. Plant Sci. 9: 251, 2018.

Liu, Y., Fang, J., Xu, F., Chu, J., Yan, C., Schlappi, M.R., Wang, Y., Chu, C.: Expression patterns of ABA and GA metabolism genes and hormone levels during rice seed development and imbibition: a comparison of dormant and non-dormant rice cultivars. - J. Genet. Genomics 41: 327-338, 2014.

Marzougui, S., Sugimoto, K., Yamanouchi, U., Shimono, M., Hoshino, T., Hori, K., Kobayashi, M., Ishiyama, K., Yano, M.: Mapping and characterization of seed dormancy QTLs

using chromosome segment substitution lines in rice. Theor. appl. Genet. 124: 893-902, 2012.

Nambara, E., Marion-Poll, A.: ABA action and interactions in seeds. - Trends Plant Sci 8: 213-217, 2003.

Ogawa, M., Hanada, A., Yamauchi, Y., Kuwahara, A., Kamiya, Y., Yamaguchi, S.: Gibberellin biosynthesis and response during Arabidopsis seed germination. - Plant Cell 15: 1591-1604, 2003.

Ogbonnaya, F.C., Imtiaz, M., Ye, G., Hearnden, P.R., Hernandez, E., Eastwood, R.F., Van Ginkel, M., Shorter, S.C., Winchester, J.M.: Genetic and QTL analyses of seed dormancy and preharvest sprouting resistance in the wheat germplasm CN10955. - Theor. appl. Genet 116: 891-902, 2008.

Ramaih, S., Guedira, M., Paulsen, G.M.: Relationship of indoleacetic acid and tryptophan to dormancy and preharvest sprouting of wheat. - Funct. Plant Biol. 30: 939-945, 2003.

Sakuma, Y., Maruyama, K., Osakabe,Y.,Qin, F., Seki, M., Shinozaki,K., Yamaguchi-Shinozaki, K.: Functional analysis of an Arabidopsis transcription factor, DREB2A, involved indrought-responsive gene expression. - Plant Cell 18: 1292-1309, 2006.

Shu, K., Zhou, W.G., Yang, W.Y.: APETALA 2-domain -containing transcription factors: focusing on abscisic acid and gibberellins antagonism. -New Phytol. 217: 977-983, 2018.

Sugimoto, K., Takeuchi, Y., Ebana, K., Miyao, A., Hirochika, H., Hara, N., Ishiyama, K., Kobayashi, M., Ban, Y., Hattori, T., Yano, M.: Molecular cloning of Sdr4, a regulator involved in seed dormancy and domestication of rice. - Proc. nat. Acad. Sci. USA 107: 5792-5797, 2010.

Wan, J., Nakazaki, T., Kawaura, K., Ikehashi, H.: Identification of marker loci for seed dormancy in rice (Oryza sativa L.). Crop Sci. 37: 1759-1763, 1997.

Wang, L., Cheng, J., Lai, Y., Du, W., Huang, X., Wang, Z., Zhang, H.: Identification of QTLs with additive, epistatic and QTL $\mathrm{x}$ development interaction effects for seed dormancy in rice. - Planta 239: 411-420, 2014.

Wang, L., Hua, D., He, J., Duan, Y., Chen, Z., Hong, X., Gong, Z.: Auxin response factor2 (ARF2) and its regulated homeodomain gene $H B 33$ mediate abscisic acid response in Arabidopsis. - PLoS Genet. 7: e1002172, 2011.

Ye, H., Feng, J., Zhang, L., Zhang, J., Mispan, M.S., Cao, Z., Beighley, D.H., Yang, J., Gu, X.Y.: Map-based cloning of seed dormancy1-2 identified a gibberellin synthesis gene regulating the development of endosperm-imposed dormancy in rice. - Plant Physiol 169: 2152-2165, 2015.

Zhao, L.F., Hu, Y.B., Chong, K., Wang,T.: ARAG1, an ABA-responsive DREB gene, plays a role in seed germination and drought tolerance of rice. - Ann. Bot. 105: 401-409, 2010.

Zhao, Y.: Auxin biosynthesis and its role in plant development. Annu. Rev. Plant Biol. 61: 49-64, 2010. 